\title{
Diagnostic Accuracy of B-Mode- and Contrast-Enhanced Ultrasound in Differentiating Malignant from Benign Pleural Effusions
}

\author{
Ehsan Safai Zadeh ${ }^{1}(\mathbb{D})$, Johanna Weide ${ }^{1}$, Christoph Frank Dietrich ${ }^{2} \mathbb{D}$, Corinna Trenker $^{3}$, \\ Andreas Rembert Koczulla ${ }^{4,5}$ and Christian Görg 1,6,*价
}

1 Interdisciplinary Center of Ultrasound Diagnostics, University Hospital Giessen and Marburg, Philipps University Marburg, Baldingerstraße, 35033 Marburg, Germany; ehsan_sz@yahoo.de (E.S.Z.); johannaweide@outlook.de (J.W.)

2 Department Allgemeine Innere Medizin (DAIM), Kliniken Hirslanden Bern, Beau Site, Salem und Permanence, 3018 Bern, Switzerland; c.f.dietrich@googlemail.com

3 Haematology, Oncology and Immunology, University Hospital Giessen and Marburg, Philipps University Marburg, Baldingerstraße, 35033 Marburg, Germany; trenker@med.uni-marburg.de

4 Institute for Pulmonary Rehabilitation Research, Schoen Klinik Berchtesgadener Land, 83471 Schoenau am Koenigssee, Germany; koczulla@med.uni-marburg.de

5 Department of Pulmonary Rehabilitation, Philipps-University of Marburg, German Center for Lung Research (DZL), 35033 Marburg, Germany

6 Gastroenterology, Endocrinology, Metabolism and Clinical Infectiology, University Hospital Giessen and Marburg, Philipp University of Marburg, Baldingerstraße, 35033 Marburg, Germany

* Correspondence: christian.goerg@uk-gm.de; Tel.: +49-6421-58-61538; Fax: +49-6421-58-62825

check for updates

Citation: Safai Zadeh, E.; Weide, J.; Dietrich, C.F.; Trenker, C.; Koczulla, A.R.; Görg, C. Diagnostic Accuracy of B-Mode- and Contrast-Enhanced Ultrasound in Differentiating Malignant from Benign Pleural Effusions. Diagnostics 2021, 11, 1293. https://doi.org/10.3390/diagnostics 11071293

Academic Editor: Ahmet F. Coskun

Received: 22 June 2021

Accepted: 14 July 2021

Published: 19 July 2021

Publisher's Note: MDPI stays neutral with regard to jurisdictional claims in published maps and institutional affiliations.

Copyright: (c) 2021 by the authors. Licensee MDPI, Basel, Switzerland. This article is an open access article distributed under the terms and conditions of the Creative Commons Attribution (CC BY) license (https:/ / creativecommons.org/licenses/by/ $4.0 /)$.

\begin{abstract}
Purpose: To evaluate the value of CEUS in differentiating malignant from benign pleural effusions (PEs). Methods: From 2008 to 2017, 83 patients with PEs of unknown cause were examined using B-mode thoracic ultrasound (B-TUS), CEUS, and cytological examination. The extent of enhancement of the pleural thickening, the presence of enhancement of septa or a solid mass within the PE, and the homogeneity of the enhancement in the associated lung consolidation, were examined. Subsequently, the diagnostic value of cytology, B-TUS, and CEUS in differentiating malignant from benign PEs was determined. Results: With CEUS, markedly enhanced pleural thickening and inhomogeneous enhanced lung consolidation were significantly more frequently associated with malignancy $(p<0.05)$. In the subgroup analysis, the use of CEUS increased the sensitivity from 69.2 to 92.3 in patients with initial negative cytology but clinical suspicion of malignant PE; it also increased the specificity from 63.0 to 90.0 , the positive predictive value from 69.2 to 92.3 , the negative predictive value from 63.0 to 90.0 , and the diagnostic accuracy from 66.7 to 87.5 , in the evaluation of PE malignancy. Conclusion: The use of clinically based B-TUS and CEUS as a complementary method to cytological evaluation may be beneficial for evaluating a PE of unknown cause. CEUS patterns of enhanced pleural thickening and inhomogeneous enhanced lung consolidation may suggest a malignant PE.
\end{abstract}

Keywords: ultrasound; CEUS; pleural effusions; cytology; diagnosis

\section{Introduction}

B-mode thoracic ultrasound (B-TUS) plays an important role in the assessment of pleural and pulmonary pathologies [1]. Due to its high sensitivity, absence of radiation exposure, and easy availability, B-TUS is the method of choice for detecting pleural effusions (PEs) [1,2]. Pleural effusions have many different causes, which may be benign or malignant [3]. For malignant PEs, the cytological examination obtained by ultrasound-guided thoracocentesis is the key to diagnosis, but it has variable sensitivity from $45.5 \%$ to $87.9 \%$, depending on the type of disease [4]. Therefore, repeated specimen collection, invasive procedures such as pleural biopsy, and, ultimately, thoracoscopy are recommended for 
improving the diagnosis if the first specimen does not provide a definitive diagnosis [5-7]. However, all these procedures are invasive and may be associated with complications, such as pneumothorax and bleeding [8]. For this reason, additional imaging methods have been introduced to clarify the cause of the PE $[9,10]$.

In computed tomography (CT), the presence of pleural thickening, nodular formations on the pleura, and an irregular pleura are considered reliable criteria for differentiating between benign and malignant PEs, and they can be helpful for directing imaging-guided pleural biopsy $[10,11]$. In a recent study, B-TUS was able to differentiate between transudate and exudative PEs [12]. It was shown that a complex septated effusion is indicative of an exudative PE [12]. Görg et al. proved in 1997 that, with B-TUS, the detection of pleural masses could be considered characteristic of a malignant effusion [9]. Despite its high specificity, B-TUS shows low sensitivity in detecting malignant PEs [13]. One of the methods to improve the sensitivity of B-TUS for the detection of malignant thoracic pathology is the use of contrast-enhanced ultrasound (CEUS) [14]. This helps to visualise different vascularisation patterns of pleural thickening, as well as focal lesions in PE-associated lung lesions; therefore, it can be helpful in differentiating between malignant and benign PEs $[15,16]$.

The aim of this study was to evaluate the value of CEUS as a complementary method to improve the diagnostic accuracy of B-TUS in combination with the cytological examination.

\section{Materials and Methods}

The study was performed between March 2008 and December 2017 in a university hospital in Germany. All study patients were referred to the Interdisciplinary Centre of Ultrasound Diagnostics for the investigation of an unclear PE and exclusion of other thoracic pathologies. The inclusion criteria were: (1) a PE of unknown cause after an initial assessment, (2) completed B-TUS and CEUS examinations, (3) simultaneous ultrasoundguided thoracocentesis with the cytological examination, and (4) clinical follow-up for 6 months. A total of 83 patients met the entry criteria and were included in the study. All specimens were cytologically examined at the local pathology institute. The final PE diagnosis was documented in the clinical records based on repetitive thoracocentesis, histological data, and a clinical course of up to 6 months. The gold standard for all computations was the final diagnosis and results of the 6-month follow-up.

This retrospective study was approved by the local ethics committee and conducted in accordance with the amended Helsinki Declaration on the ethical principles for medical research involving human subjects. Informed consent was obtained from each patient for the ultrasound examinations.

\subsection{Ultrasound Examinations}

The B-TUS examinations were performed with an ACUSON SEQUOIA 512 GI ultrasound machine (Siemens, Erlangen, Germany). A 4C1 curved array transducer with frequencies between 3.5 and $4.5 \mathrm{MHz}$ was used. The CEUS examinations were performed with the same transducer in $1.5 \mathrm{MHz}$ contrast-specific mode according to the EFSUMB guidelines [17]. A bolus injection of $2.4 \mathrm{~mL}$ of SonoVue ${ }^{\circledR}$ (Bracco Imaging S.p.A., Milan, Italy) contrast medium was given via peripheral venous access, followed by $10 \mathrm{~mL} \mathrm{NaCl}$ $0.9 \%$. During the first $30 \mathrm{~s}$, PEs and thoracic pathologies were continuously examined. Subsequently, several short examinations in $30 \mathrm{~s}$ intervals, up to $3 \mathrm{~min}$, were conducted. The pathologies presented in CEUS imaging were saved as images. All the patients were examined in an upright sitting position. All the ultrasound examinations were performed standardised and prospective by a German Society for Ultrasound in Medicine (DEGUM)qualified Level-III examiner (C.G.). The ultrasound data were obtained during general clinical procedures and according to the hospital's guidelines.

The following B-TUS data and CEUS parameters were retrospectively analysed by two independent, experienced investigators (E.S., C.G.). In the event of a discrepancy between 
the two investigators, the final decision was made by a third experienced investigator (C.T.). Cohen's kappa statistics were applied to measure interrater reliability.

\subsection{B-Mode Lung Ultrasound Parameters}

1. The presence of pleural thickening $(>3 \mathrm{~mm})$, classified as nodular or flat thickening.

2. The pleural-effusion volume, classified as $>1000 \mathrm{~mL}$ or $<1000 \mathrm{~mL}$. The volume was measured using the following formula: Pleural effusion volume $(\mathrm{mL})=70 \times$ (basal lung-diaphragm distance $(\mathrm{cm})+$ max. effusion height $(\mathrm{cm}))$ [18].

3. The presence of a septated PE or a solid mass within the PE.

4. The homogeneity of the associated pulmonary consolidation, classified as homogeneous or inhomogeneous.

\subsection{Contrast-Enhanced Ultrasound Parameters}

1. The extent of enhancement of the pleural thickening in the arterial phase was classified as reduced or absent and marked enhancement. Splenic enhancement was used as an in vivo reference for comparison [16,19].

2. The presence of enhancement of septa or a solid mass within the PE [16].

3. The homogeneity of enhancement in the associated lung consolidation was classified as homogeneous or inhomogeneous. Inhomogeneous enhancement was defined as a perfused lesion with coexisting non-perfused or reduced perfused areas [15,20].

\subsection{Statistical Analysis}

Statistical evaluation was conducted on the categorical variable with Fisher's exact test and on the continuous data with Mann-Whitney tests. Cohen's kappa statistics were applied to measure interrater reliability, and a $p$-value $<0.05$ was defined as significant. In addition, sensitivity, specificity, positive and negative predictive values, and diagnostic accuracy were evaluated for the cytological examination and the pathological ultrasound findings associated with malignancy.

\section{Results}

\subsection{Characteristics of the Participants}

Of the 83 patients, 60 were men and 23 were women. The average age was 62.2 years, with a range of 21-91. The final clinical diagnosis was malignant PE in 42/83 (50.6\%) of the patients and benign PE in $41 / 83(49.4 \%)$. There was no significant difference between patients with benign and malignant PEs in terms of age or gender $(p>0.05)$.

The final causes of PEs in all the patients are shown in Table 1.

Table 1. Causes of PEs in $n=83$ patients.

\begin{tabular}{|c|c|c|c|}
\hline Cause of Benign PE & $\begin{array}{c}\text { Number }(\%) \\
\text { of Patients }\end{array}$ & Cause of Malignant PE & $\begin{array}{c}\text { Number (\%) } \\
\text { of Patients }\end{array}$ \\
\hline Parapneumonic PE & $18(43.9)$ & Pulmonary metastasis of solid carcinomas & $17(40.5)$ \\
\hline Pleura empyema & $7(17.1)$ & Primary bronchial carcinoma & $17(40.5)$ \\
\hline Congestive heart failure & $9(22.0)$ & Pleural mesothelioma & $4(9.5)$ \\
\hline Pulmonary artery embolism & $3(7.3)$ & Lung infiltration by malignant lymphoma & $2(4.8)$ \\
\hline Non-infectious granulomatous diseases & $2(4.9)$ & Pulmonary metastasis of sarcoma & $2(4.8)$ \\
\hline Liver cirrhosis & $1(2.4)$ & - & - \\
\hline IgG4-related pleuritis & $1(2.4)$ & - & - \\
\hline Total number of patients with benign PE & $41(100)$ & Total number of patients with malignant PE & $42(100)$ \\
\hline
\end{tabular}




\subsection{Initial Cytopathological Data of the Patients}

On the initial cytopathological examination, malignant cells in the effusions were detectable in $n=29(34.9 \%)$ of the patients, and these effusions were classified as malignant PEs. In $54(65.1 \%)$ of the patients, an initial cytopathological examination showed no malignant cells. Regarding the final cause of PE, the initial cytopathological examination showed a sensitivity of $69.0 \%$, specificity of $100 \%$, positive predictive value of $100 \%$, negative predictive value of $75.9 \%$, and diagnostic accuracy of $84.3 \%$ for identifying a malignant PE.

Among the patients with negative initial cytology, 24/54 (44.4\%) cases had a clinical suspicion of malignant PE, despite this initial result. In the re-evaluation of clinical data after 6 months, the PE was secondarily classified as malignant in 13/24 (54.2\%) of the patients through a histopathological examination after either a pleural $(7 / 13 ; 53.8 \%)$ or a pulmonary biopsy $(6 / 13 ; 46.2 \%)$. In $11 / 24(45.8 \%)$ of the patients with an initial benign cytology but suspected malignancy, a final benign PE was diagnosed by a clinical follow-up and confirmation of a benign cause (six parapneumonic, four congestive heart disease, and one non-infectious granulomatous disease). In 30/54 (55.6\%) of the patients, the PE was initially classified as benign, in accordance with their "benign" clinical background. In the re-evaluation of clinical data after 6 months, all these PEs were confirmed to be truly benign. Figure 1 presents the work-up to the final diagnosis of PE with respect to the initial cytopathological results, clinical background, and follow-up.

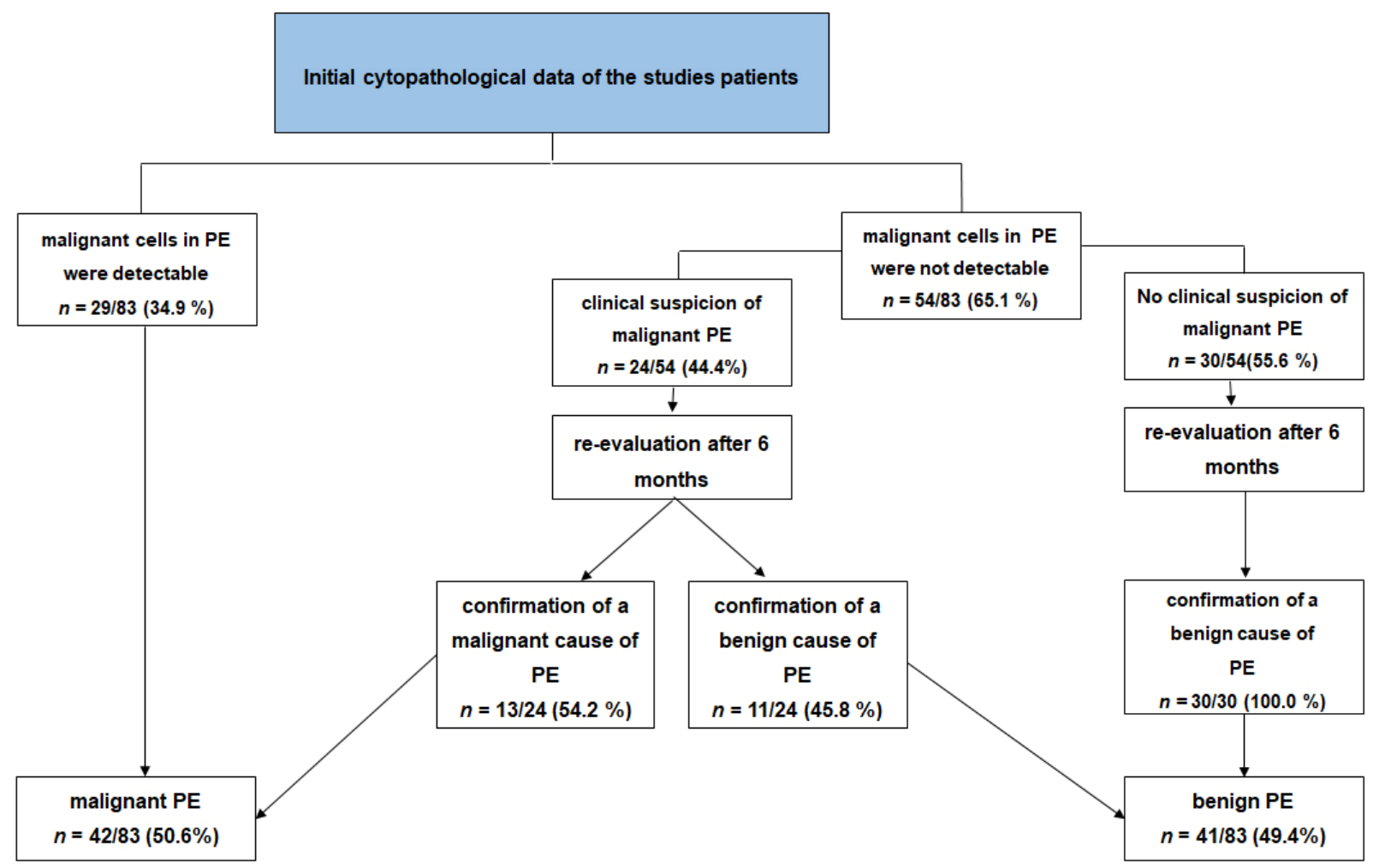

Figure 1. Work-up to the final diagnosis of PE in 83 patients with respect to the initial cytopathological results, clinical background, and follow-up.

\subsection{Ultrasound Data}

There was a good agreement between the examiners for the B-TUS and CEUS parameters $($ Cohen's kappa $=0.78)$. 


\subsection{B-Mode Thoracic Ultrasound}

The diagnostic data from the B-TUS examination are summarised in Table 2.

In terms of the presence of a septated PE or a solid mass in the PE and inhomogeneous lung consolidation in the B-TUS images, there were no significant differences between patients with a malignant PE and those with a benign PE ( $p>0.05$, Fisher's exact test). The presence of a PE with a volume $>1000 \mathrm{~mL}$ and pleural thickening was significantly more frequently associated with malignancy $(p<0.05$, Fisher's exact test). Among the patients with pleural thickening $(28 / 83 ; 33.7 \%), 12 / 28(42.9 \%)$ revealed nodular (Figure 2A,C), and $16 / 28(57.1 \%)$ had flat thickening of the pleura (Figure 3A,C).

In those with nodular pleural thickening, 11/12 (91.7\%) cases had malignant PEs, and those with flat pleural thickening had malignant PEs in 9/16 (56.3\%) of the cases.
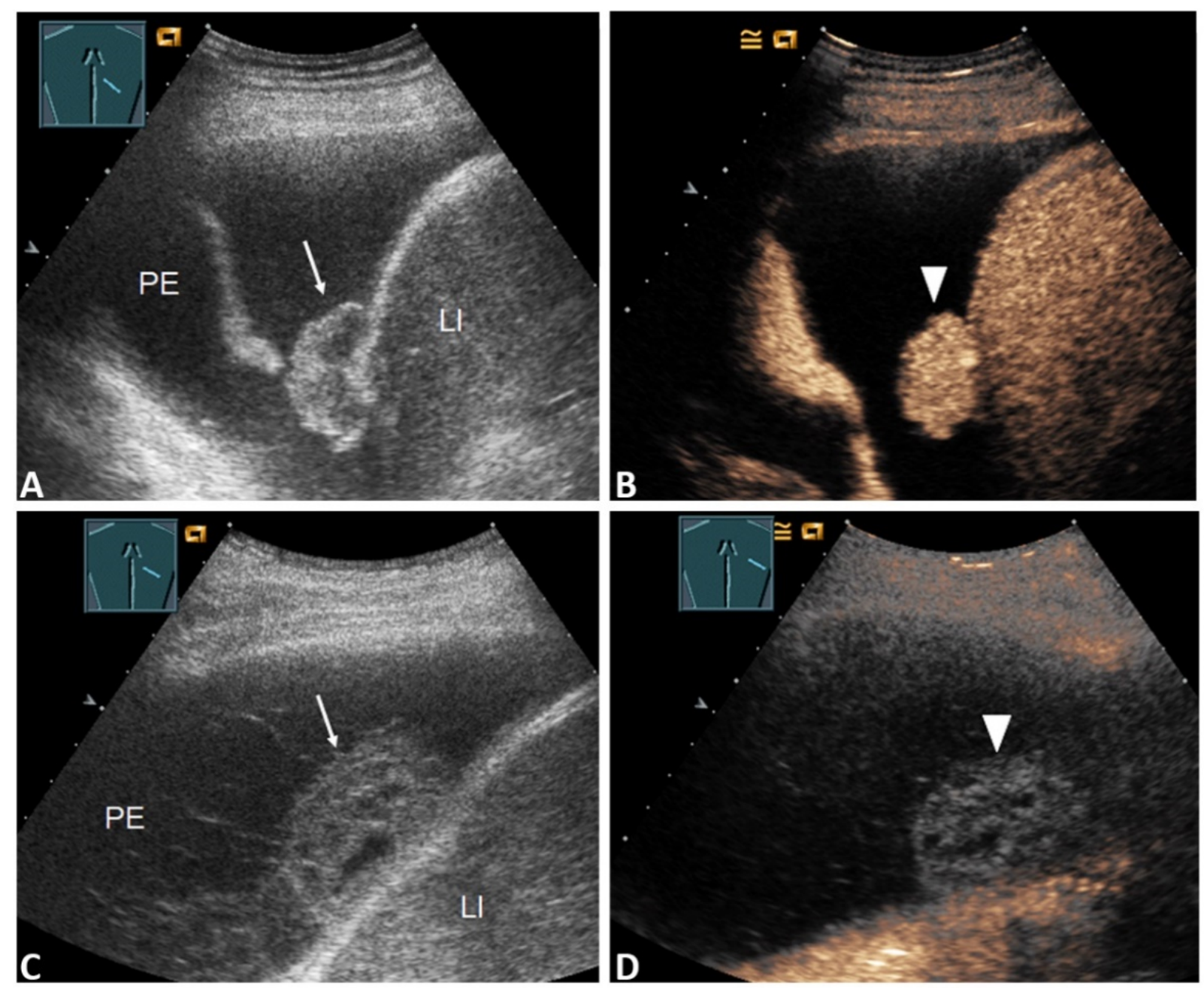

Figure 2. (A) 59-year-old patient with a right-sided PE. The effusion cytology was negative. B-TUS shows conspicuous nodular pleural thickening (arrow); (B) In the CEUS images, the nodular pleural thickening shows marked enhancement, such as in metastasis (stick). The final diagnosis was a malignant PE based on histologically confirmed pulmonary sarcoma. $\mathrm{LI}=$ Liver. (C) 64-year-old patient with fever, dyspnea, and a right PE. Effusion cytology was negative. B-US shows a septated effusion with a nodular formation on the diaphragm (arrow); (D) CEUS shows nodular pleural thickening with no enhancement, similar to fibrin bodies (sticks). The final diagnosis was a benign parapneumonic PE. LI = Liver. 
Table 2. B-TUS data of the $n=83$ patients, subdivided between final malignant and benign PE.

\begin{tabular}{|c|c|c|c|c|}
\hline Variable & $\begin{array}{l}\text { All Patients } \\
\quad n=83\end{array}$ & $\begin{array}{l}\text { Malignant PE } \\
\quad n=42\end{array}$ & $\begin{array}{c}\text { Benign PE } \\
n=41\end{array}$ & $p$-Value \\
\hline \multicolumn{5}{|c|}{ Pathological ultrasound findings with B-TUS } \\
\hline Pleural thickening & $28(33.7 \%)$ & $20(47.6 \%)$ & $8(19.5 \%)$ & \multirow{2}{*}{0.010 * } \\
\hline No pleural thickening & $55(66.3 \%)$ & $22(52.4 \%)$ & $33(80.5 \%)$ & \\
\hline PE volume $\leq 1000$ & $51(61.4 \%)$ & $21(50.0 \%)$ & $30(73.2 \%)$ & \multirow{2}{*}{$0.042 *$} \\
\hline PE volume $>1000$ & $32(38.6 \%)$ & $21(50.0 \%)$ & $11(26.8 \%)$ & \\
\hline Septa or solid formation in PE & $24(28.9 \%)$ & $8(19.0 \%)$ & $16(39.0 \%)$ & \multirow{2}{*}{0.055 * } \\
\hline No septa or solid formation in PE & $59(71.1 \%)$ & $34(81.0 \%)$ & $25(61.0 \%)$ & \\
\hline Inhomogeneous lung consolidation & $12(14.5 \%)$ & $9(21.4 \%)$ & $3(7.3 \%)$ & \multirow{2}{*}{$0.116^{*}$} \\
\hline Homogeneous lung consolidation & $71(85.5 \%)$ & $33(78.6 \%)$ & $38(92.7 \%)$ & \\
\hline
\end{tabular}

The values are indicated as number (\%). A $p$-value $<0.05$ was defined as significant. *: Fisher's exact test.
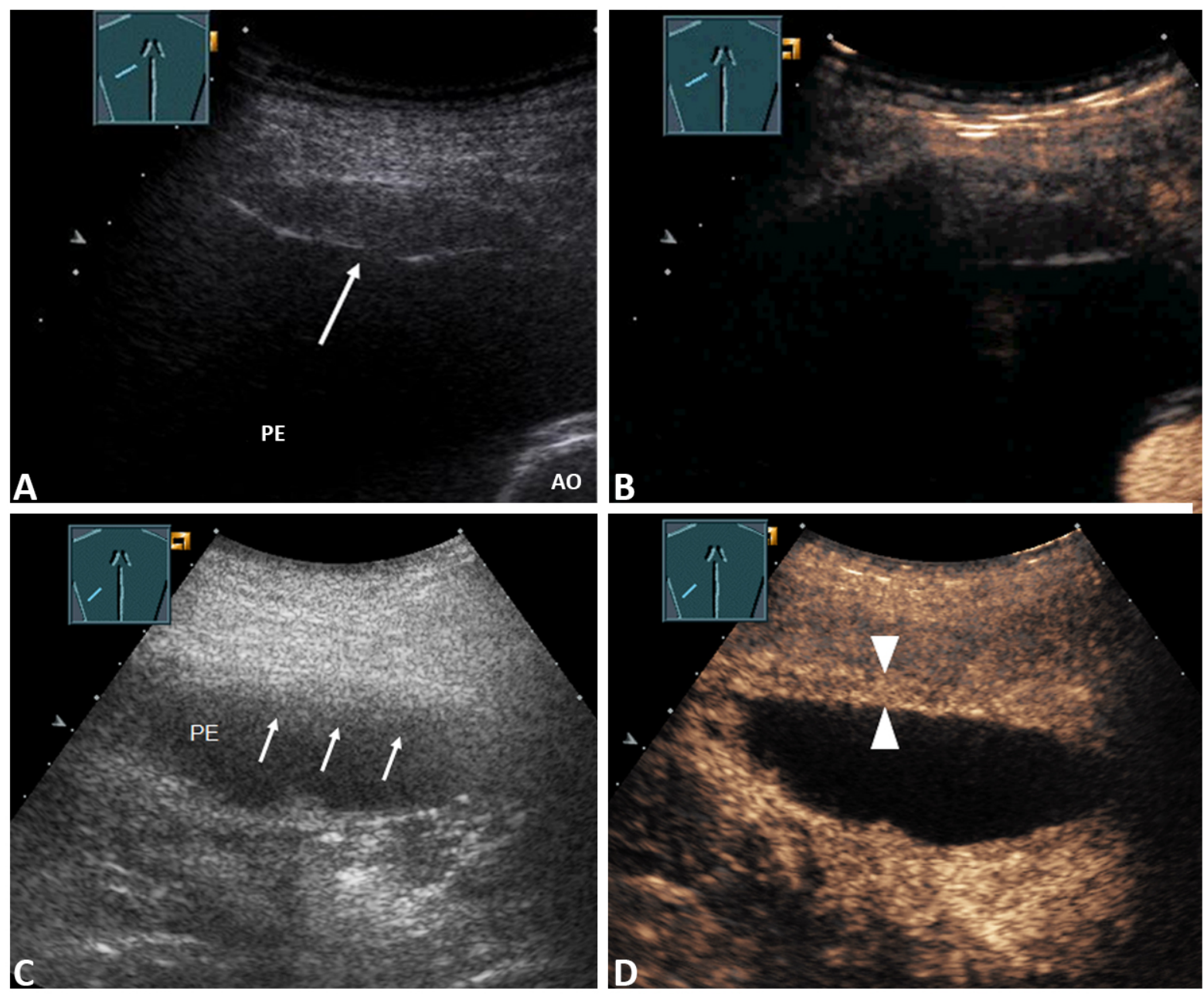

Figure 3. (A) 74-year-old patient with decompensated heart failure and a right-sided PE. The effusion cytology is negative. Pleural thickening is conspicuous in the B-TUS images (arrow); (B) CEUS shows pleural thickening with no enhancement; the final diagnosis was a benign PE with pleural thickening, based on histologically confirmed fibrinous pleuritis. AO = Aorta. (C) 75-year-old patient with chronic obstructive pulmonary disease, fever, and a left-sided narrow PE. The effusion cytology was negative. B-US shows conspicuous fuzzy pleural thickening (arrows); (D) CEUS reveals sharp pleural thickening with marked enhancement (sticks). The final diagnosis was a benign PE due to empyema. 


\subsection{Contrast-Enhanced Thoracic Ultrasound}

In total, using CEUS, a pleural thickening was detected in $n=33 / 83$ (39.8\%) study patients, and no pleural thickening was detected in $n=50 / 83(60.2 \%)$. In $n=27 / 33$ $(81.1 \%)$ patients, a pleural thickening with marked enhancement was seen. Furthermore, in $n=6 / 33(18.9 \%)$ patients, a pleural thickening with reduced or absent enhancement was observed. Using CEUS, in total, five more flat pleural thickenings and 13 more inhomogeneous pulmonary consolidations were detected in comparison with B-TUS. Regarding nodular pleural thickening or effusion pathologies (septa), there was no difference in the visualisation of pathologic structures between B-TUS and CEUS. Further details concerning the diagnostic data from the CEUS examination are summarised in Table 3.

Table 3. CEUS data of the $n=83$ patients, subdivided between final malignant and benign PE.

\begin{tabular}{|c|c|c|c|c|}
\hline Variable & $\begin{array}{l}\text { All Patients } \\
\quad n=83\end{array}$ & $\begin{array}{l}\text { Malignant PE } \\
\quad n=42\end{array}$ & $\begin{array}{l}\text { Benign PE } \\
\quad n=41\end{array}$ & $p$-Value \\
\hline \multicolumn{5}{|c|}{ Pathological ultrasound findings with CEUS } \\
\hline Pleural thickening with marked enhancement (Figures 2B and 3D) & $27(32.5 \%)$ & $20(47.6 \%)$ & $7(17.1 \%)$ & \multirow[b]{2}{*}{$0.005 *$} \\
\hline $\begin{array}{l}\text { Pleural thickening with absent or reduced enhancement or no } \\
\text { pleural thickening (Figure 3B) }\end{array}$ & $56(67.5 \%)$ & $22(52.4 \%)$ & $34(82.9 \%)$ & \\
\hline Enhancement of septa or solid formation (Figure 4D) & $2(2.4 \%)$ & $2(4.8 \%)$ & $0(0.0 \%)$ & \multirow[b]{2}{*}{$0.494 *$} \\
\hline No enhancement of septa or solid formation (Figure 5C) & $81(97.6 \%)$ & $40(95.2 \%)$ & $41(100.0 \%)$ & \\
\hline Inhomogeneous enhancement of lung consolidation (Figure 5B-D) & $25(30.1 \%)$ & $19(45.2 \%)$ & $6(14.6 \%)$ & \multirow{2}{*}{0.004 * } \\
\hline Homogeneous enhancement of lung consolidation & $58(69.9 \%)$ & $23(54.8 \%)$ & $35(85.4 \%)$ & \\
\hline
\end{tabular}

The values are indicated as number $(\%)$. A $p$-value $<0.05$ was defined as significant. *: Fisher's exact test.

Among those with a marked enhancement of pleural thickening $(27 / 83 ; 32.5 \%)$, $n=11 / 27(40.7 \%)$ patients revealed nodular, and $n=16(59.3 \%)$ had flat thickening of the pleura. Of the patients with nodular pleural thickening and marked enhancement, all $11 / 11(100.0 \%)$ cases had a final malignant PE (Figure 2A,B). Of those with flat pleural thickening, $n=9 / 16(56.3 \%)$ had a final malignant PE. In all patients with benign PEs, flat pleural thickening, and marked enhancement $(7 / 7 ; 100 \%)$, the underlying disease was pleural empyema, as shown in Figure 3D. In those with pleural thickening and absent or reduced enhancement, $n=5 / 6(83 \%)$ patients had a benign final diagnosis (Figure $3 \mathrm{~B}$ ), and in $n=1 / 6(17 \%)$ a malignant PE was cytologically confirmed.

In total, using CEUS, an inhomogeneous enhancement of the lung consolidation was detected in $n=25 / 83$ (30.1\%) study patients. Inhomogeneous enhancement of the lung consolidation with a final malignant PE was found in 19 patients (Figure 5B).

The causes were primary bronchial carcinoma in 10/19 (52.6\%) patients, pulmonary metastasis of solid carcinomas in 6/19 (31.6\%) patients (Figure 4B), and pleural mesothelioma in 3/19 (15.8\%) patients.

Inhomogeneous enhancement of the lung consolidation with a benign PE was found in six patients. The causes were an inflammatory process (parapneumonic, non-infectious granulomatous diseases and IgG4-related pleurisy) for 3/6 (50\%) of the patients and pulmonary artery embolism for the other $3 / 6$ (50\%; see Figure 5D).

From the CEUS images, marked enhancement of the pleural thickening (Figure 2B) and inhomogeneous enhancement of the lung consolidation (Figure 5B) were significantly more frequently associated with a malignant PE $(p<0.05$, Fisher's exact test).

Furthermore, there were no significant differences between patients with a malignant PE and those with a benign PE in terms of the presence of septal enhancement or a solid mass in the PE. However, all the PEs with the presence of septa enhancement were malignant $(2 / 2 ; 100 \%)$, as shown in Figure 4D and Table 3.

Data from the diagnostic accuracy of the initial cytopathological examination, B-TUS, and CEUS for all the patients in terms of sensitivity, specificity, and positive and negative predictive value are summarised in Table 4. 

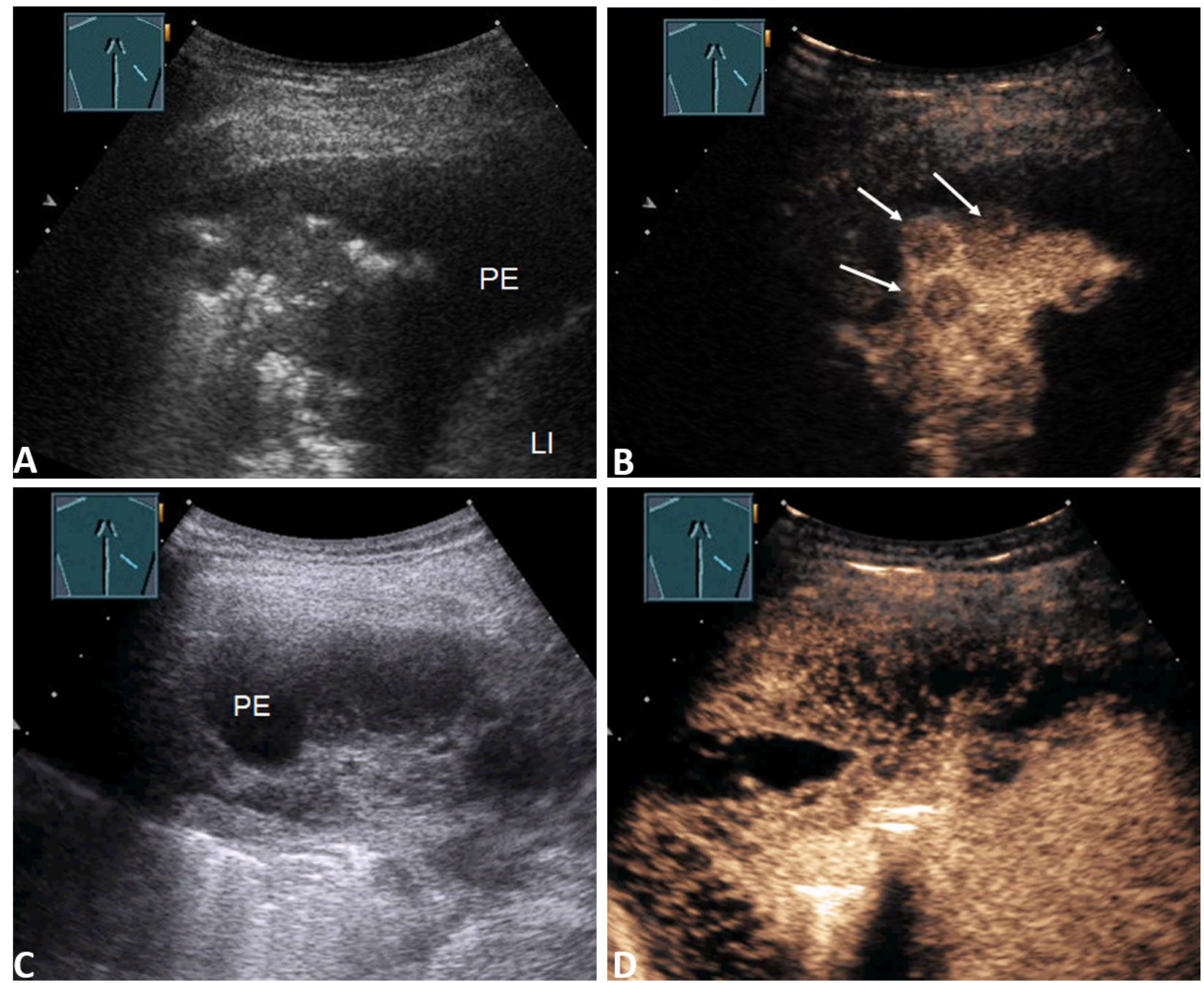

Figure 4. (A) 55-year-old patient with a right-sided PE. Effusion cytology was negative. B-TUS shows inhomogeneous lung consolidation; (B) In the CEUS images, the lung consolidation shows hypoechoic round lesions such as metastases (arrows). The final diagnosis was a malignant PE based on histologically confirmed metastatic adenocarcinoma of the oesophagus. LI = Liver. (C) 55-year-old patient with a right-sided PE. Effusion cytology was positive. B-TUS shows a polyseptated effusion; (D) CEUS shows marked enhancement of the septa. The final diagnosis was a malignant effusion due to mantle cell lymphoma with lung involvement.

Table 4. Diagnostic performance of initial cytopathological examination, B-US, and CEUS for evaluating PE malignancy in $n=83$ patients.

\begin{tabular}{|c|c|c|c|c|c|}
\hline Variable & $\begin{array}{l}\text { Sensitivity } \\
(\%)\end{array}$ & $\begin{array}{l}\text { Specificity } \\
(\%)\end{array}$ & PPV (\%) & NPV (\%) & $\begin{array}{r}\text { Diagnostic } \\
\text { Accuracy }(\%)\end{array}$ \\
\hline Initial cytopathological examination & 69.0 & 100.0 & 100.0 & 75.9 & 84.3 \\
\hline $\begin{array}{c}\text { B-TUS data associated with malignancy } \\
\text { (volume of } \mathrm{PE}>1000 \mathrm{~mL} \text {, pleural thickening) }\end{array}$ & 69.1 & 58.5 & 63.0 & 64.7 & 63.9 \\
\hline $\begin{array}{l}\text { CEUS data associated with malignancy (pleural thickening } \\
\text { with marked enhancement and inhomogeneous } \\
\text { enhancement of pulmonary consolidations) }\end{array}$ & 73.8 & 70.7 & 72.1 & 72.5 & 72.3 \\
\hline B-TUS and CEUS data associated with malignancy & 88.1 & 46.3 & 62.7 & 79.2 & 67.5 \\
\hline
\end{tabular}

The values are indicated as number (\%). PPV = positive predictive value; NPV = negative predictive value. 

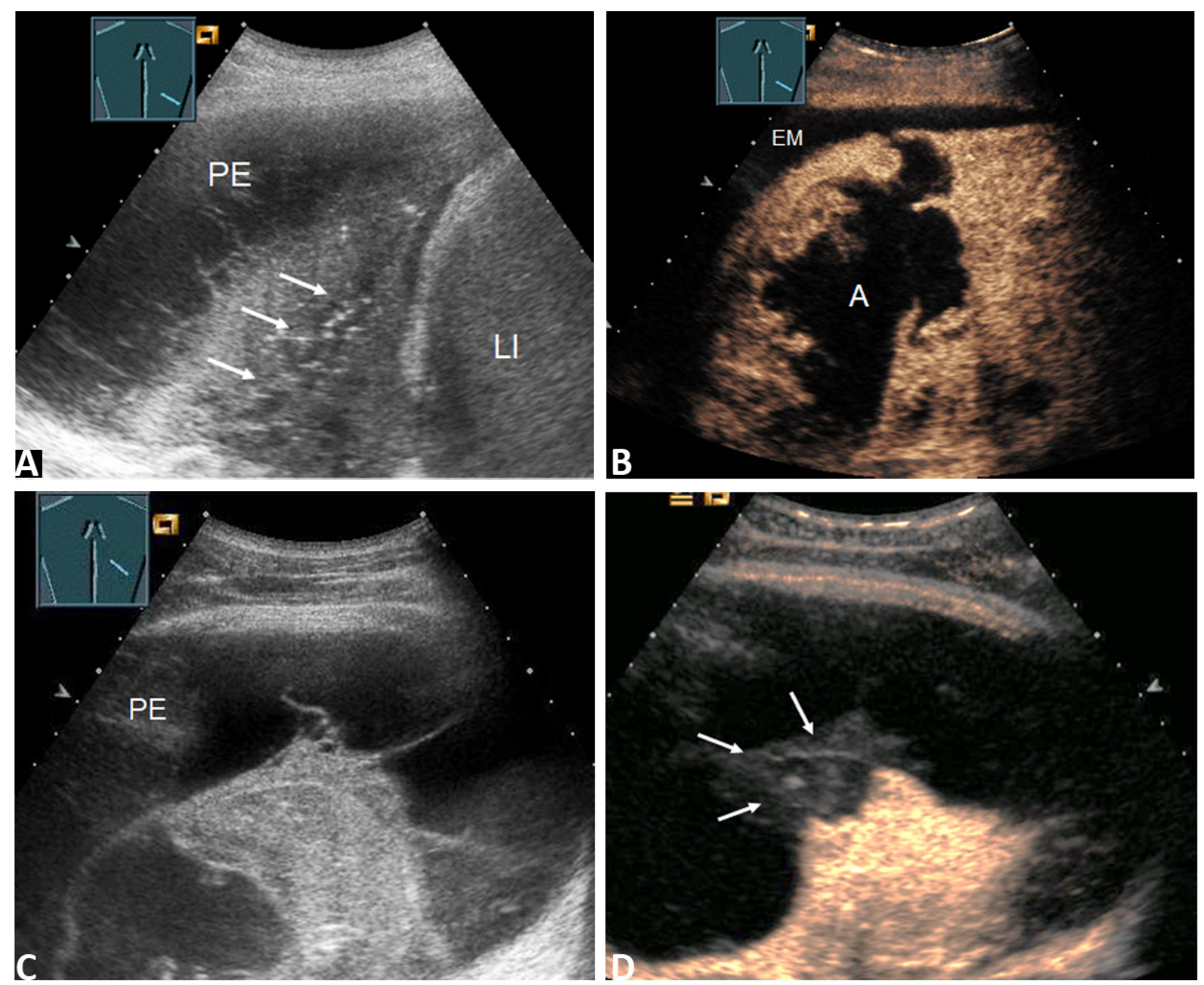

Figure 5. (A) 68-year-old patient with a right lateral PE. Effusion cytology was negative. B-TUS shows a PE with septa and inhomogeneous lung consolidation (arrows); (B) In the CEUS images, the lung consolidation shows a large anechoic liquidation, such as in cases of abscess or necrosis. The final diagnosis was a malignant PE due to central non-small cell lung carcinoma (NSCLC) with consecutive lung abscess (A) and pleural empyema (EM). LI = Liver. (C) 27-year-old patient with fever and a right-sided PE. Effusion cytology was negative B-TUS shows a PE with septation and lung consolidation; (D) CEUS shows consolidation with inhomogeneous enhancement and a peripheral non-enhanced wedge-shaped area similar to a pulmonary infarction (arrows). The final diagnosis was a benign PE with a pulmonary artery embolism morphologically confirmed through CT pulmonary angiography and histologically confirmed infarct pneumonia.

\subsection{Negative Cytology and Clinical Suspicion}

The 24 patients with an initial negative cytopathological examination but clinical suspicion of malignant effusion were investigated in a subgroup analysis (Figure 1).

Within this subgroup, the presence of one or more parameters associated with malignancy in the B-TUS images showed a sensitivity of $69.2 \%$, specificity of $63.4 \%$, positive predictive value of $69.2 \%$, negative predictive value of $63.4 \%$, and diagnostic accuracy of $66.7 \%$ for the identification of a malignant PE (Table 5).

The presence of one or more parameters associated with malignancy in the CEUS images had a sensitivity of $92.3 \%$, specificity of $90.0 \%$, positive predictive value of $92.3 \%$, negative predictive value of $90.0 \%$, and diagnostic accuracy of $87.5 \%$ for the identification of a malignant PE (Table 5). 
Table 5. Diagnostic performance of B-TUS and CEUS for evaluating PE malignancy in a subgroup of $n=24$ patients with initial negative cytology and clinical suspicion of malignant PE.

\begin{tabular}{|c|c|c|c|c|c|}
\hline Variable & $\begin{array}{l}\text { Sensitivity } \\
(\%)\end{array}$ & $\begin{array}{l}\text { Specificity } \\
\quad(\%)\end{array}$ & PPV (\%) & NPV (\%) & $\begin{array}{r}\text { Diagnostic } \\
\text { Accuracy }(\%)\end{array}$ \\
\hline $\begin{array}{l}\text { B-TUS data associated with malignancy } \\
\text { (volume of } \mathrm{PE}>1000 \mathrm{~mL} \text {, pleural thickening) }\end{array}$ & 69.2 & 63.4 & 69.2 & 63.4 & 66.7 \\
\hline $\begin{array}{l}\text { CEUS data associated with malignancy (pleural thickening } \\
\text { with marked and inhomogeneous enhancement of } \\
\text { pulmonary consolidations) }\end{array}$ & 92.3 & 90.0 & 92.3 & 90.0 & 87.5 \\
\hline B-TUS and CEUS data associated with malignancy & 92.3 & 54.6 & 70.6 & 85.7 & 75.0 \\
\hline
\end{tabular}

The values are indicated as number (\%). PPV: positive predictive value; NPV: negative predictive value.

\section{Discussion}

For the evaluation of unclear PE, contrast-enhanced $\mathrm{CT}$ is recommended as the method of choice in the guidelines from the British Thoracic Society [21]. In addition to contrastenhanced CT, B-TUS is widely used as a cost-effective method without radiation exposure for the evaluation of PE $[1,9,15]$. However, only limited data are available regarding the diagnostic performance of CEUS as an additional imaging modality in the diagnosis of unclear PE [16].

In this retrospective study, we investigated the diagnostic accuracy of B-TUS and CEUS for 83 patients with PEs of unknown cause. Furthermore, we investigated the diagnostic value of B-TUS and CEUS for evaluating PE malignancy in all the patients, as well as in a subgroup of 24 with initial negative cytology and clinical suspicion of malignant PE.

Regarding B-TUS patterns, we found that the detection of a PE volume $>1000 \mathrm{~mL}$ and pleural thickening was significantly more often associated with malignant effusion. From the CEUS images, pleural thickening with marked contrast enhancement and inhomogeneous enhancement of the lung consolidation was significantly more often associated with malignancy. In this study, the PEs were malignant in all 11/11 (100\%) cases with nodular pleural thickening and marked enhancement. Interestingly, $6 / 11(54.5 \%)$ of the effusions in these patients were classified as benign in the initial cytopathological examination. Based on these findings, nodular pleural thickening with marked enhancement should be considered malignant, even if there is no indication of malignancy in cytopathological examinations.

For all the patients in the study, the diagnostic accuracy was $63.9 \%$ for B-TUS and $72.3 \%$ for CEUS in terms of evaluating malignancy. The diagnostic performance of B-TUS and CEUS in the present study compared with the findings of previously performed B-TUS and contrast-enhanced CT studies is summarised in Table 6.

Table 6. Diagnostic performance of B-TUS, CEUS, and CECT for evaluating PE malignancy.

\begin{tabular}{ccccccccc}
\hline $\begin{array}{c}\text { Imaging } \\
\text { Modality }\end{array}$ & Cases & Year & Author & $\begin{array}{c}\text { Sensitivity } \\
\mathbf{( \% )}\end{array}$ & $\begin{array}{c}\text { Specificity } \\
\mathbf{( \% )}\end{array}$ & $\begin{array}{c}\text { PPV } \\
(\mathbf{\%})\end{array}$ & $\begin{array}{c}\text { NPV } \\
(\mathbf{\%})\end{array}$ & $\begin{array}{c}\text { Diagnostic } \\
\text { Accuracy (\%) }\end{array}$ \\
\hline B-TUS & 52 & 2008 & Qureshi et al. [22] & 73 & 100 & 100 & 79 & NS \\
\hline B-TUS & 133 & 2014 & Bugalho et al. [23] & 80.3 & 83.6 & 82.8 & 81.2 & 81.9 \\
\hline B-TUS & 83 & 2021 & Present study & 69.1 & 58.5 & 63.0 & 64.7 & 63.9 \\
\hline CEUS & 83 & 2021 & Present study & 73.8 & 70.7 & 72.1 & 72.5 & 72.3 \\
\hline CECT & 40 & 2000 & Traill et al. [10] & 87.0 & 100 & 100 & 67.0 & 90.0 \\
\hline CECT & 343 & 2015 & Porcel et al. [24] & 74.0 & 92.0 & NS & NS & NS \\
\hline
\end{tabular}

B-TUS: B-mode thoracic ultrasound; CECT: contrast-enhanced computed tomography; CEUS: contrast-enhanced ultrasound; NPV: negative predictive value; NS: not specified; PPV: positive predictive value. 
The higher diagnostic performance in the two previously performed B-TUS studies may be due to the inclusion criteria, the differences in patient spectrums, and the examination criteria in these studies. In both studies, only patients with suspicion of malignant effusion were included [22,23]. Furthermore, both studies considered the presence of hepatic metastases as an indication of malignant PE $[22,23]$. In the study by Qureshi et al., all patients with clinical or radiological features of empyema were excluded [22]. This may have resulted in selection bias and could explain the higher specificity and positive predictive value compared with the findings of the current study.

The two previously performed contrast-enhanced CT studies showed higher sensitivity and specificity compared with CEUS in the diagnosis of malignant PE [10,24]. Compared with ultrasound, CT provides a better overview and can visualise more areas of the thorax. In ultrasound, due to air and the bony structures of the thorax, only approximately $70 \%$ of the pleura can be visualised and examined [25]. This could be the reason for the higher diagnostic performance of CT compared with CEUS.

In contrast to the results for the patients in the overall study, the subgroup analysis of those with an initial negative cytology and clinical suspicion of malignant PE revealed a better diagnostic value for CEUS compared to B-TUS regarding the evaluation of malignancy. The use of CEUS increased the sensitivity in the subgroup from 69.2 to 92.3 , the specificity from 63.0 to 90.0 , the positive predictive value from 69.2 to 92.3 , the negative predictive value from 63.0 to 90.0 , and the diagnostic accuracy from 66.7 to 87.5 for the evaluation of PE malignancy. In this group, CEUS showed high diagnostic performance and could be considered to be a useful imaging modality.

Pleural thickening occurs in both benign and malignant diseases [16], and it may be the result of an inflammatory process, which is indicated with a marked enhancement of pleural thickening in the CEUS images [26,27]. In this study, the causes of all pleural thickenings with marked enhancement were malignant or inflammatory processes due to pleural empyema. This finding can be used in combination with clinical suspicion to improve the identification of malignant PEs in patients with initially negative cytological results. A further value of CEUS is the identification of disturbed perfusion of associated lung consolidation $[20,28,29]$. Inhomogeneous perfusion seen in the CEUS can be found in various pathological conditions, including peripheral pulmonary infarction, peripheral pulmonary granulomatous lesions [28], chronic pneumonia-associated lung abscess, and tumour-associated necrosis or vascularisation disturbance in the consolidated lung [15,29]. PE-associated lung consolidations with peripheral wedge-shaped non-perfused areas are highly suggestive of infarction in patients with pulmonary embolic disease [20,30]. Pneumonia associated lung lesions may indicate lung abscesses with secondary development of parapneumonic empyema [15]. On the other hand, centrally located round lesions in the consolidated lung may indicate lung metastasis or necrosis [15]. These findings can be used in combination with clinical suspicion to improve the identification of the causes of PE. It should be highlighted that sonographic diagnosis is fundamentally based on clinical signs and symptoms. Finally, it should be mentioned that, in all detected pleural or parenchymatous lesions, CEUS-guided biopsy with histological examination is the method of choice to achieve the final diagnosis [31,32].

There are some limitations to this study. This study is limited by its retrospective nature. Furthermore, ultrasound examination is generally characterised by high interobserver variability. We could not blind the examiners to the study group, and blind interpretation of the ultrasound data by the ultrasound investigator was not possible. Furthermore, the study was performed on patients who were referred to the Interdisciplinary Centre of Ultrasound Diagnostics for the investigation of an unclear PE and exclusion of other thoracic pathologies and were investigated standardised by a single DEGUM-qualified Level-III examiner. Therefore, selection bias cannot be excluded. Finally, a pleural or a pulmonary biopsy with histological validation was not performed on all study patients. 


\section{Interpretation}

The findings of this study show that using clinically based B-TUS and CEUS as complementary methods to cytopathological examinations are beneficial for evaluating PEs of unknown causes. Furthermore, CEUS patterns of pleural thickening with marked enhancement and an inhomogeneous perfusion pattern of lung consolidation may suggest a malignant PE. However, for a definitive diagnosis of PE malignancy, repetitive cytology, clinical work-up, and, if necessary, a pleural or pulmonary histopathological examination is warranted.

Author Contributions: E.S.Z. and C.G. designed the study and were guarantors of the paper. E.S.Z. evaluated and interpreted the data and wrote the manuscript based on the support and comments of all other authors. All authors participated in the data preparation, literature search, and interpretation of results. C.G., E.S.Z., C.F.D., A.R.K., J.W. and C.T. reviewed the manuscript. All authors have read and agreed to the published version of the manuscript.

Funding: This research received no funding.

Institutional Review Board Statement: The study was conducted according to the guidelines of the Declaration of Helsinki and approved by the Ethics Committee of Philipps University Marburg (protocol code: EK_MR_26-06-20_görg and date of approval: 26.06.2020).

Informed Consent Statement: Informed consent was obtained from all subjects involved in the study for ultrasound examinations.

Data Availability Statement: The data presented in this study are available on request from the corresponding author.

Acknowledgments: The work of Ehsan Safai Zadeh is funded in part by a research grant from the Anneliese Pohl Foundation (Anneliese Pohl Stiftung), and we gratefully acknowledge this support.

Conflicts of Interest: C. Görg received funding from Bracco Imaging. Bracco Imaging supported CEUS workshops at the University Hospital Marburg.

\section{References}

1. Dietrich, C.F.; Mathis, G.; Cui, X.-W. Ultrasound of the pleurae and lungs. Ultrasound Med. Biol. 2015, 41, 351-365. [CrossRef]

2. Feller-Kopman, D. Ultrasound-Guided Thoracentesis. Chest 2006, 129, 1709-1714. [CrossRef] [PubMed]

3. Jany, B.; Welte, T. Pleural Effusion in Adults-Etiology, Diagnosis, and Treatment. Dtsch. Aerzteblatt Online 2019, 116, 377-386. [CrossRef] [PubMed]

4. Loveland, P.; Christie, M.; Hammerschlag, G.; Irving, L.; Steinfort, D. Diagnostic yield of pleural fluid cytology in malignant effusions: An Australian tertiary centre experience. Intern. Med. J. 2018, 48, 1318-1324. [CrossRef] [PubMed]

5. Bielsa, S.; Panadés, M.J.; Egido, R. Rentabilidad del estudio citológico del líquido pleural en el derrame maligno. Anales de medicina interna. Madr. Spain 1984 2008, 25, 173-177.

6. Rodríguez-Panadero, F.; Janssen, J.P.; Astoul, P. Thoracoscopy: General overview and place in the diagnosis and management of pleural effusion. Eur. Respir. J. 2006, 28, 409-422. [CrossRef] [PubMed]

7. Pandit, S.; Chaudhuri, A.D.; Datta, S.B.S.; Dey, A.; Bhanja, P. Role of pleural biopsy in etiological diagnosis of pleural effusion. Lung India 2010, 27, 202-204. [CrossRef] [PubMed]

8. Cantey, E.P.; Walter, J.M.; Corbridge, T. Complications of thoracentesis: Incidence, risk factors, and strategies for prevention. Curr. Opin. Pulm. Med. 2016, 22, 378-385. [CrossRef]

9. Görg, C.; Restrepo, I.; Schwerk, W.B. Sonography of malignant pleural effusion. Eur. Radiol. 1997, 7, 1195-1198. [CrossRef]

10. Traill, Z.C.; Davies, R.J.; Gleeson, F.V. Thoracic Computed Tomography in Patients with Suspected Malignant Pleural Effusions. Clin. Radiol. 2001, 56, 193-196. [CrossRef]

11. Maskell, N.A.; Gleeson, F.V.; Davies, R.J.O. Standard pleural biopsy versus CT-guided cutting-needle biopsy for diagno-sis of malignant disease in pleural effusions: A randomised controlled trial. Lancet 2003, 361, 1326-1330. [CrossRef]

12. Shkolnik, B.; Judson, M.A.; Austin, A. Diagnostic Accuracy of Thoracic Ultrasonography to Differentiate Tran-sudative From Exudative Pleural Effusion. Chest 2020, 158, 692-697. [CrossRef] [PubMed]

13. Shiroshita, A.; Nozaki, S.; Tanaka, Y.; Luo, Y.; Kataoka, Y. Thoracic ultrasound for malignant pleural effusion: A systematic review and meta-analysis. ERJ Open Res. 2020, 6, 464-2020. [CrossRef] [PubMed]

14. Görg, C.; Bert, T.; Kring, R.; Dempfle, A. Transcutaneous contrast enhanced sonography of the chest for evaluation of pleural based pulmonary lesions: Experience in 137 patients. Ultraschall der Med. Eur. J. Ultrasound 2006, 27, 437-444. [CrossRef]

15. Mathis, G. (Ed.) Bildatlas der Lungensonographie. 6., Vollständig Überarbeitete und Aktualisierte Auflage; Springer: Berlin/Heidelberg, Germany, 2016. 
16. Safai Zadeh, E.; Görg, C.; Dietrich, C.F. Contrast-Enhanced Ultrasound for Evaluation of Pleural Effusion: A Pictorial Essay. J. ultrasound Med. Off. J. Am. Inst. Ultrasound Med. 2021. online ahead of print. [CrossRef] [PubMed]

17. Sidhu, P.S.; Cantisani, V.; Dietrich, C.F. Die EFSUMB-Leitlinien und Empfehlungen für den klinischen Einsatz des kontrastverstärkten Ultraschalls (CEUS) bei nicht-hepatischen Anwendungen: Update 2017 (Langversion). Ultraschall Med. Stuttg. Ger. 1980 2018, 39, e2-e44.

18. Goecke, W.; Schwerk, W.B. Die Real-Time Sonographie in der Diagnostik von Pleuraergüssen; Springer Science and Business Media LLC: Berlin/Heidelberg, Germany, 1990; pp. 385-387.

19. Lim, A.K.P.; Patel, N.; Eckersley, R.J. Evidence for spleen-specific uptake of a microbubble contrast agent: A quantitative study in healthy volunteers. Radiology 2004, 231, 785-788. [CrossRef]

20. Trenker, C.; Dohse, M.; Ramaswamy, A.; Michel, C.; Görg, C. Histological validation of pulmonary infarction detected with contrast-enhanced ultrasound in patients with negative computed tomography pulmonary angiogram: A case series. J. Clin. Ultrasound 2019, 47, 461-465. [CrossRef]

21. Hooper, C.; Lee, Y.C.G.; Maskell, N. Investigation of a unilateral pleural effusion in adults: British Thoracic Society pleural disease guideline 2010. Thorax 2010, 65 (Suppl. 2), ii4-ii17. [CrossRef]

22. Qureshi, N.R.; Rahman, N.M.; Gleeson, F. Thoracic ultrasound in the diagnosis of malignant pleural effusion. Thorax 2009, 64, 139-143. [CrossRef]

23. Bugalho, A.; Ferreira, D.; Dias, S.S.; Schuhmann, M.; Branco, J.C.; Gomes, M.J.M.; Eberhardt, R. The Diagnostic Value of Transthoracic Ultrasonographic Features in Predicting Malignancy in Undiagnosed Pleural Effusions: A Prospective Observational Study. Respiration 2014, 87, 270-278. [CrossRef]

24. Porcel, J.M.; Pardina, M.; Bielsa, S.; González, A.; Light, R.W. Derivation and validation of a CT scan scoring system for discriminating malignant from benign pleural effusions. Chest 2015, 147, 513-519. [CrossRef]

25. Mathis, G. Thoraxsonography—Part II: Peripheral pulmonary consolidation. Ultrasound Med. Biol. 1997, 23, 1141-1153. [CrossRef]

26. Saito, A.; Hakamata, Y.; Yamada, Y.; Sunohara, M.; Tarui, M.; Murano, Y.; Mitani, A.; Tanaka, K.; Nagase, T.; Yanagimoto, S. Pleural thickening on screening chest X-rays: A single institutional study. Respir. Res. 2019, 20, 1-7. [CrossRef]

27. Sack, U.; Hoffmann, M.; Zhao, X.J.; Chan, K.S.; Hui, D.S.C.; Gosse, H.; Engelmann, L.; Schauer, J.; Emmrich, F.; Hoheisel, G. Vascular endothelial growth factor in pleural effusions of different origin. Eur. Respir. J. 2005, 25, 600-604. [CrossRef]

28. Safai Zadeh, E.; Keber, C.U.; Dietrich, C.F. Perfusion Patterns of Peripheral Pulmonary Granulomatous Lesions Using ContrastEnhanced Ultrasound (CEUS) and Their Correlation with Immunohistochemically Detected Vascularization Patterns. J. ultrasound Med. Off. J. Am. Inst. Ultrasound Med. 2021. online ahead of print. [CrossRef]

29. Safai Zadeh, E.; Beutel, B.; Dietrich, C.F. Perfusion patterns of peripheral pulmonary lesions in COVID-19 pa-tients using contrast-enhanced ultrasound (CEUS): A case series. J. Ultrasound Med. Off. J. Am. Inst. Ultrasound Med. 2021. online ahead of print. [CrossRef]

30. Bartelt, S.; Trenker, C.; Görg, C. Contrast-enhanced ultrasound of embolic consolidations in patients with pulmonary embolism: A pilot study. J. Clin. Ultrasound JCU 2016, 44, 129-135. [CrossRef]

31. Cao, B.-S.; Wu, J.-H.; Li, X.-L. Sonographically guided transthoracic biopsy of peripheral lung and mediastinal lesions: Role of contrast-enhanced sonography. J. Ultrasound Med. Off. J. Am. Inst. Ultrasound Med. 2011, 30, 1479-1490. [CrossRef] [PubMed]

32. Fu, Y.; Zhang, Y.-Y.; Cui, L.-G.; Tan, S.; Sun, Y. Ultrasound-Guided Biopsy of Pleural-Based Pulmonary Lesions by Injection of Contrast-Enhancing Drugs. Front. Pharmacol. 2019, 10, 960. [CrossRef] [PubMed] 